\title{
Síndrome de ovario poliquístico (SO P) y embarazo: Experiencia clínica
}

\author{
Ana Claudia Villarroel ${ }^{1}$, Bárbara Echiburú2a, \\ Virginia Riesco $0^{1 b}$, Manuel Maliqueo $2 c$, Michael Cárcamo ${ }^{2 d}$, \\ Catalina Hitschfeld², Fernando Sánchez ${ }^{2}$, \\ María Paz del Solar ${ }^{2}$ y Teresa Sir-Petermann².
}

\section{Polycystic ovary syndrome and pregnancy: Clinical experience}

Background: Polycystic ovary syndrome (PCOS) is an endocrine metabolic dysfunction closely associated with insulin resistance and obesity, which predisposes to pregnancy complications. Aim: To report a prospective clinical experience in PCOS patients who became pregnant after diet, exercise and metformin treatment intervention, and were followed up during the whole pregnancy. Patients and Methods: Seventy pregnant PCOS (PPCOS) women and forty normal pregnant (NP) women of similar age and with singleton pregnancies were included in the study. During gestational ages 10-16 and 22-28 weeks, $a$ 2h, $75 \mathrm{~g}$ oral glucose tolerance test (OGTT) was performed with measurement of glucose and insulin in each sample. Results: No differences were found in duration of gestation, weight gain during pregnancy, or systolic and diastolic blood pressure between PPCOS and NP women. There were significant differences in body mass index (BMI) at the initiation and in the third trimester of pregnancy between both groups. The incidence of gestational diabetes was significantly higher $(p<0.01)$ in the PCOS group (35.2\%) compared to the control group (5.0\%). The prevalence of small for gestational age (SGA) infants tended to be higher ( $p=0.09)$ in the PCOS group. During pregnancy, $2 \mathrm{~h}$ glucose and insulin were significantly bigher in PPCOS than in NP women. Conclusions: PCOS mothers showed a higher prevalence of gestational diabetes and SGA newborns, which cannot be attributed to the weight gain during pregnancy, and seems to be more related to the BMI at the initiation of pregnancy, and to the PCOS condition of the mother (Rev Méd Chile 2007; 135: 1530-38).

(Key words: Diabetes gestational; Polycystic ovary syndrome; Pregnancy complications)

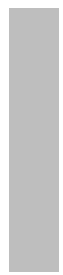

Recibido el 24 de noviembre, 2006. Aceptado el 19 de julio, 2007.

Financiado por el Proyecto FONDECYT 1030487.

${ }^{1}$ Unidad de Diabetes y Nutrición y ${ }^{2}$ Unidad de Endocrinología, Departamento de Medicina, Campus Occidente, Universidad de Chile. Hospital San Juan de Dios. Santiago de Chile.

angeniero en Biotecnología Molecular.

bNutricionista, M.Sc.

'Tecnólogo Médico, M.Sc.

${ }^{\mathrm{d}}$ Matrón.

Correspondencia a: Dra. T. Sir-Petermann. Las Palmeras 299, Interior Quinta Normal. Casilla 33052, Correo 33. Santiago, Chile. Tel: 56-2-6814676. Fax: 56-2-6816693.

E mail: tsir@med.uchile.cl 
$\mathrm{E}^{1}$ síndrome de ovario poliquístico (SOP) es una disfunción endocrino-metabólica altamente prevalente (5\%-10\%) y es la causa más común de hiperandrogenismo y disfunción ovulatoria en mujeres premenopáusicas. Su etiología es incierta y se encuentra en estrecha asociación con la insulino resistencia (IR) y la diabetes tipo 2, las que suelen presentarse precozmente ${ }^{1-3}$. Debido a la alta prevalencia de obesidad e IR, las mujeres con SOP tienen un mayor riesgo de presentar patologías del embarazo ${ }^{4}$, una mala historia obstétrica y el nacimiento de un niño pequeño para la edad gestacional (PEG) o macrosómico 5,6 . Además, de acuerdo a nuestra experiencia ${ }^{7}$ y a estudios publicados posteriormente ${ }^{8}$, se ha establecido que las mujeres con SOP presentan altos niveles de insulina y andrógenos durante el embarazo, en comparación a controles sanos, lo que podría incidir en el peso de nacimiento de sus hijos y en la mayor prevalencia de patologías del embarazo ${ }^{6,7}$.

Por otro lado, la obesidad y la IR juegan un papel relevante en la anovulación crónica y en la infertilidad de estas pacientes, lo que significa que una disminución del peso corporal y de la IR serían el primer acercamiento terapéutico en pacientes con SOP insulino resistentes que desean fertilidad. El cambio del estilo de vida asociado al uso de un sensibilizante a la acción de la insulina, como la metformina, sería beneficioso en recuperar la fertilidad en más de $80 \%$ de las mujeres que presentan una pérdida de peso de al menos 5\%9-13.

El objetivo del presente estudio es mostrar nuestra experiencia clínica prospectiva en mujeres con SOP que consultaron por deseo de fertilidad en el laboratorio de Endocrinología y Metabolismo de la Facultad de Medicina Occidente de la Universidad de Chile; en el período 2000-2005 y que lograron embarazo de término después de un esquema terapéutico consistente en dieta, ejercicio y metformina; y que fueron seguidas durante todo el embarazo y hasta el nacimiento de sus hijos.

\section{PACIENTES Y MÉTODOS}

Mujeres con SOP: De 150 pacientes con diagnóstico de SOP y deseos de fertilidad que consultaron durante los años 2000 a 2005 en el policlínico docente del Laboratorio de Endocrinología y Metabolismo de la Facultad de Medicina Occidente; 120 se embarazaron y 70 aceptaron participar en el estudio. Se incluyeron embarazadas con SOP (EcSOP) normoglicémicas con IR, entre 20 y 35 años de edad, con embarazo único de término. El diagnóstico de SOP se hizo de acuerdo al criterio de Rotterdam ${ }^{14}$. Se incluyeron pacientes con historia de irregularidad menstrual, ecotomografía sugerente de SOP, ausencia de patología adrenal o hipofisiaria y presencia de hiperandrogenemia.

Mujeres sin SOP: Se estudiaron 40 embarazadas sin SOP (EsSOP) de edad similar a las pacientes con SOP y embarazo único de término, que tenían antecedentes de ciclos menstruales regulares, 28 a 32 días, ausencia de manifestaciones de hiperandrogenismo, que no estaban recibiendo ningún tipo de medicamento y no presentaban antecedentes de patologías crónicas. Las EsSOP fueron reclutadas a partir de la semana 12 de gestación desde los policlínicos de atención primaria de la misma área geográfica de las pacientes con SOP, durante el mismo período de tiempo.

Se excluyeron mujeres de ambos grupos con antecedentes de tabaquismo, alcoholismo y abuso de drogas. Previo al estudio, se obtuvo el consentimiento informado de todas las mujeres. Este estudio fue aprobado por el Comité de Ética de la Investigación en Seres Humanos de la Facultad de Medicina de la Universidad de Chile el Comité Ético-Científico (CEC) del Servicio de Salud Metropolitano Central y el Comité de Ética Científica del Servicio de Salud Metropolitano Occidente.

Con el fin de contar con valores de glicemia e insulinemia de mujeres normales no embarazadas, se construyó un grupo de referencia con los datos obtenidos de 80 mujeres sanas de edad similar a las pacientes con SOP.

Metodología. Previo al embarazo, se consignaron medidas antropométricas (peso, talla, perímetro de cintura y cadera) y se realizó una encuesta alimentaria y de actividad física en todas las pacientes. Se practicó una prueba de tolerancia a la glucosa oral (PTGO) con $75 \mathrm{~g}$ de glucosa y medición de glicemia e insulinemia. En la muestra basal se determinó además la concentración de andrógenos y lípidos. Se realizó una ecotomogra- 
fía ginecológica transvaginal para detectar morfología de ovario poliquístico ${ }^{15}$.

Con el fin de lograr embarazo, las mujeres con SOP ingresaron a un programa de tratamiento consistente en ejercicio físico regular, dieta de bajo índice glicémico con ajuste de calorías según peso corporal y metformina. La dieta consistió en el aporte de $30 \mathrm{Kcal} / \mathrm{Kg}$ de peso a las mujeres de peso normal y $25 \mathrm{Kcal} / \mathrm{Kg}$ de peso a aquellas con obesidad o sobrepeso. El 55\% de las calorías totales correspondía a hidratos de carbono y se privilegiaron las harinas integrales y los granos, restringiendo al máximo los carbohidratos de absorción rápida, $15 \%$ de proteína y 25\%-30\% de grasas con selección de ácidos grasos mono y poliinsaturados y con aumento de ácidos grasos omega-3. Además, a todas ellas se les indicó ejercicio aeróbico programado que consistió en 30 minutos al día de caminata enérgica, bicicleta o natación. Las pacientes recibieron metformina en fórmula estándar entre 500 a 2.000 mg al día según peso corporal, tolerancia digestiva y niveles de insulina, desde el momento en que se les diagnosticó IR hasta que se estableció el diagnóstico de embarazo, momento en el cual la metformina fue suspendida paulatinamente.

Durante el embarazo se adecuó la ingesta calórica agregando $300 \mathrm{Kcal}$ por día correspondientes al estado de gravidez y se mantuvo la actividad física dependiendo de las condiciones obstétricas de cada paciente.

Protocolo de estudio. Ambos grupos de embarazadas fueron controladas en sus respectivos policlínicos maternales. Se consignó duración de la gestación, índice de masa corporal (IMC) inicial e IMC del tercer trimestre, ganancia de peso durante el embarazo y presión arterial. Además, se registraron patologías del embarazo y características de los recién nacidos. Los datos de peso e IMC inicial corresponden a aquellos que presentaron las mujeres al momento en que se diagnosticó el embarazo (peso inicio de embarazo). El IMC en el tercer trimestre fue calculado a partir del peso durante las semanas 22 a 28 de embarazo. La ganancia de peso fue calculada como la diferencia entre el peso final del embarazo y el peso al inicio. Las patologías del embarazo se consignaron a partir de la semana 12 y hasta el final de la gestación. En ambos grupos de embarazadas se realizó una prueba de tolerancia a la glucosa oral (PTGO), con 75 g de glucosa entre la semana 1016 y 22-28 de gestación. Se determinó glucosa e insulina en la muestra basal, a los 60 y 120 minutos post carga de glucosa. El diagnosticó de diabetes mellitus gestacional (DMG) se hizo de acuerdo al criterio del American College of Obstetrics and Gynecology 200116, que es similar al propuesto por el Ministerio de Salud (MINSAL): glicemia basal mayor a $100 \mathrm{mg} / \mathrm{dl}$ (en dos oportunidades) y mayor a $140 \mathrm{mg} / \mathrm{dl}$ a las $2 \mathrm{~h}$ postcarga de glucosa.

Mediciones: Se determinó glucosa sérica por el método de glucosa oxidasa (Instrumento fotométrico 4010; Roche, Basilea, Suiza). El coeficiente de variación de este método fue menor al $2 \%$. La insulina en el suero se determinó por RIA (Diagnostic Systems Laboratories, Inc. Texas, USA), los coeficientes de variación intra e inter ensayo fueron de 5 y $8 \%$, respectivamente.

Análisis estadístico: Los valores se expresan como promedio \pm desviación estándar $(\mathrm{X} \pm \mathrm{DE})$ en las Tablas 1 y 3. Con el fin de entregar valores de referencia para embarazadas chilenas normales, los datos de la Tabla 2 se expresan como promedio y rango. La distribución normal de los datos se evaluó mediante la prueba de Kolmogorov-Smirnov. Las diferencias entre EcSOP y EsSOP fueron analizadas mediante la prueba de t' de Student, mientras que la variable peso intra-grupo fue evaluada por ANOVA para muestras repetidas. Las diferencias entre datos categóricos se evaluó mediante la prueba de $\chi^{2}$ y test exacto de Fisher. Todos los análisis estadísticos fueron realizados usando el programa estadístico STATA 7.0. Un valor de p menor de 0,05 fue considerado estadísticamente significativo.

\section{Resultados}

En la Tabla 1 se resumen las características clínicas y las variables antropométricas de las EsSOP y EcSOP. La talla en ambos grupos fue similar, mientras que el peso y el IMC inicial fueron mayores en las EcSOP que en las EsSOP. Por su parte, el IMC en el tercer trimestre del embarazo también fue mayor en las EcSOP que en las 
Tabla 1. Características clínicas de las embarazadas sin SO P (ESSO P) y con síndrome de ovario poliquístico (ECSO P)

\begin{tabular}{|lccc|}
\hline & $\begin{array}{c}\text { EsSOP } \\
(\mathrm{n}=40)\end{array}$ & $\begin{array}{c}\text { EcSOP } \\
(\mathrm{n}=70)\end{array}$ & $\mathrm{p}$ \\
\hline Edad (años) & $27,2 \pm 4,9$ & $27,5 \pm 4,0$ & 0,782 \\
Talla (ms) & $1,6 \pm 0,05$ & $1,6 \pm 0,07$ & 0,090 \\
Peso inicial (k) & $60,1 \pm 8,4$ & $72,6 \pm 12,3$ & 0,001 \\
IMC inicial $\left(\mathrm{k} / \mathrm{m}^{2}\right)$ & $24,4 \pm 3,3$ & $28,3 \pm 5,7$ & 0,001 \\
IMC en el tercer trimestre $\left(\mathrm{k} / \mathrm{m}^{2}\right)$ & $26,4 \pm 3,0$ & $30,8 \pm 4,6$ & 0,001 \\
Ganancia de peso durante el embarazo (kg) & $10,7 \pm 5,2$ & $12,1 \pm 6,2$ & 0,296 \\
Presión sistólica (mmHg) & $114,2 \pm 6,4$ & $117,0 \pm 11,2$ & 0,165 \\
Presión diastólica (mmHg) & $69,9 \pm 6,0$ & $73,9 \pm 9,7$ & 0,067 \\
Síndrome hipertensivo del embarazo (\%) & 2,5 & 7,1 & 0,410 \\
Diabetes gestacional (\%) & 5,0 & 34,3 & 0,001 \\
\hline
\end{tabular}

Los valores son expresados como promedios \pm desviación estándar.

EsSOP. No obstante, la ganancia de peso en estas pacientes fue similar al de las embarazadas normales. La presión sistólica y diastólica no fue estadísticamente diferente entre los grupos. La prevalencia de diabetes gestacional fue significativamente mayor en las EcSOP en comparación a las EsSOP.
La evolución del peso corporal en las mujeres con SOP durante el embarazo se muestra en la Figura 1. Al momento de la primera consulta (previo al embarazo), el peso de las pacientes fue de 76,5 $\pm 10,5 \mathrm{Kg}$. Como se desprende de la figura hubo una baja de peso de alrededor de $5 \%$ entre la primera consulta (momento en que se diagnosticó

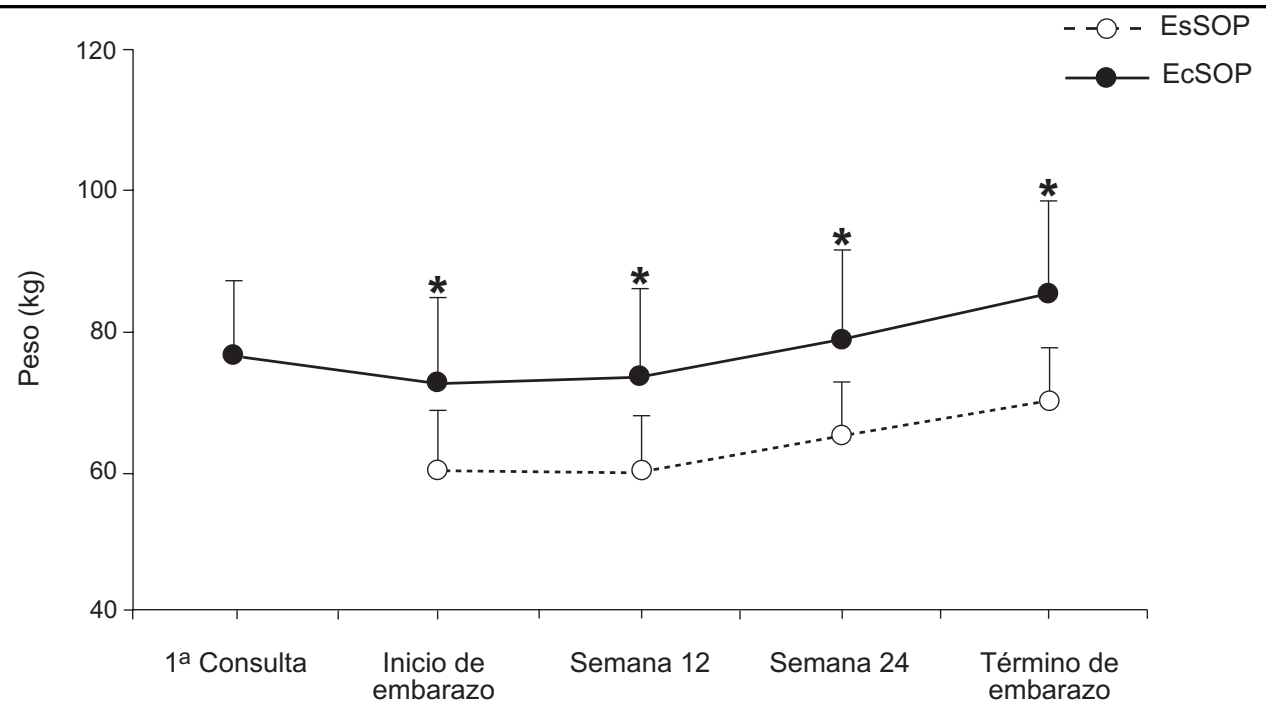

Figura 1. Evolución del peso corporal de las mujeres embarazadas con SOP (EcSOP) y embarazadas sin SOP (EsSOP) antes y durante el embarazo. Los valores se expresan como promedio \pm desviación estándar. *p $<0,05$ entre EsSOP vs EcSOP. 
Tabla 2. Concentración de glucosa e insulina basal y post carga en embarazadas sin SOP (ESSO P) y embarazadas con síndrome de ovario poliquístico (ECSO P) en la semana de gestación 10-16 y 22-28

\begin{tabular}{|lccc|}
\hline & $\begin{array}{c}\text { EsSOP } \\
(\mathrm{n}=40)\end{array}$ & $\begin{array}{c}\text { EcSOP } \\
(\mathrm{n}=70)\end{array}$ & $\mathrm{p}$ \\
\hline $\begin{array}{l}\text { Semana } 10-16 \\
\text { Basal }\end{array}$ & & & \\
$\begin{array}{l}\text { Glucosa }(\mathrm{mg} / \mathrm{dl}) \\
\text { Insulina }(\mu \mathrm{IU} / \mathrm{ml})\end{array}$ & $79,2(59,8-104,4)$ & $77,8(56,0-110,0)$ & 0,532 \\
$2-h$ & $11,9(3,0-59,4)$ & $16,0(3,0-68,9)$ & 0,122 \\
Glucosa $(\mathrm{mg} / \mathrm{dl})$ & $90,9(60,0-142,0)$ & $108,1(64,5-162,2)$ & 0,001 \\
Insulina $(\mu \mathrm{IU} / \mathrm{ml})$ & $56,0(4,0-194,2)$ & $105,4(7,2-292,7)$ & 0,001 \\
\hline Semana $22-28$ & & & \\
Basal & & $75,1(50,5-108,0)$ & 0,638 \\
Glucosa $(\mathrm{mg} / \mathrm{dl})$ & $76,2(57,0-110,0)$ & $14,4(4,4-41,5)$ & 0,844 \\
Insulina $(\mu \mathrm{IU} / \mathrm{ml})$ & $15,0(3,9-76,8)$ & $112,9(63,5-170,5)$ & 0,001 \\
$2-h$ & & $106,5(25,0-223,1)$ & 0,021 \\
Glucosa $(\mathrm{mg} / \mathrm{dl})$ & $95,9(73,0-132,0)$ & & \\
Insulina $(\mu \mathrm{IU} / \mathrm{ml})$ & $73,6(4,0-289,0)$ & & \\
\hline
\end{tabular}

Los valores son expresados como promedios y rango.

p-value entre EsSOP vs EcSOP.

Tabla 3. Características de los recién nacidos de embarazadas sin SO P (EsSOP) y con síndrome de ovario poliquístico (EcSO P).

\begin{tabular}{|lccc|}
\hline & $\begin{array}{c}\text { EsSOP } \\
(\mathrm{n}=40)\end{array}$ & $\begin{array}{c}\text { EcSOP } \\
(\mathrm{n}=70)\end{array}$ & $\mathrm{p}$ \\
\hline Edad gestacional (sem) & $39,3 \pm 1,1$ & $38,9 \pm 1,1$ & 0,163 \\
Peso (grs) & $3354,6 \pm 396,3$ & $3415,2 \pm 458,2$ & 0,565 \\
Talla (cms) & $50,0 \pm 1,8$ & $49,9 \pm 1,9$ & 0,806 \\
Circunferencia craneana (cms) & $35,1 \pm 0,9$ & $34,7 \pm 1,2$ & 0,254 \\
PEG (\%) & 2,5 & 12,9 & 0,090 \\
GEG (\%) & 12,5 & 14,2 & 0,890 \\
\hline
\end{tabular}

Los valores son expresados como promedios \pm desviación estándar.

p-value entre EsSOP vs EcSOP.

la condición de SOP e IR) y el peso de inicio del embarazo $(72,6 \pm 12,3 \mathrm{Kg})$. Posteriormente, a partir del primer trimestre (semana 12 de embarazo), se observó la esperada ganancia de peso al avanzar la gestación. Las glicemias e insulinemias basales y postcarga de las mujeres con SOP antes del embarazo, fueron superiores a los valores de referencia obtenidos en mujeres normales de edad comparable. Glicemia basal: $87,8(59,0-172,0)$ vs 80,4 (59,0-107,0); $\mathrm{p}=0,005$. Glicemia postcarga:
$117,7(65,0-269,0)$ vs 89,1 (60,0-143,0); $\mathrm{p}=0,001$. Insulinemia basal: 23,1 (4,2-76,0) vs 11,1 (3,0$51,8) ; \mathrm{p}=0,001$. Insulinemia postcarga: 130,4 (11,9454,1) vs 43,2 (3,0-195,0); $\mathrm{p}=0,001$. La Tabla 2 muestra las glicemias e insulinemias basales y postcarga en ambos grupos de embarazadas en los 2 períodos estudiados. No hubo diferencias significativas en las semanas de gestación en las cuales se realizaron las PTGO en los dos períodos estudiados (EsSOP: 12,5 \pm 1,0 y 24,2 $\pm 0,9$ semanas 
vs EcSOP: $12,7 \pm 1,2$ y $24,1 \pm 0,5$ semanas). Durante la gestación, las glicemias basales de las embarazadas con SOP fueron comparables a los valores de glicemia de las EsSOP, mientras que los valores postcarga de glucosa fueron más altos en las EcSOP en ambos períodos. Por su parte, las insulinemias mostraron el mismo patrón descrito para las glicemias, siendo los valores postcarga siempre mayores en las EcSOP. La Tabla 3 muestra las características clínicas de los recién nacidos de las EsSOP y EcSOP. El peso y talla de nacimiento, edad gestacional y circunferencia craneana fueron comparables entre los niños nacidos de madres con SOP y los nacidos de madres sin SOP. Sólo 2 niños PEG nacieron de madres con SOP que presentaron preeclampsia. La incidencia de niños PEG en el grupo de madres con SOP no se asoció a la paridad, a la talla de la madre ni a diabetes gestacional. Sin embargo, cuando se analizaron los niños por curva de peso según Juez et $\mathrm{al}^{17}$, la prevalencia de recién nacidos pequeños para la edad gestacional (PEG) tendió a ser mayor en el grupo de madres con SOP $(p=0,090)$.

\section{DisCUSIÓN}

El presente estudio muestra una experiencia clínica prospectiva en mujeres con SOP que lograron embarazo con un esquema terapéutico simple y que fueron seguidas hasta el nacimiento de sus hijos.

El éxito de nuestro esquema terapéutico enfocado en combatir la IR, es concordante con estudios previos que establecen que medidas no farmacológicas (dieta y ejercicio) asociadas a una droga insulinosensibilizante como la metformina, permite recuperar la tasa de ovulación en un alto porcentaje de las mujeres con $\mathrm{SOP}^{18-22}$. Lo anterior se evidenció además, en la baja de peso que experimentaron las pacientes entre la instauración del tratamiento y el inicio del embarazo. Si bien la utilidad de la metformina con fines reproductivos está ampliamente demostrada, el tipo de dieta recomendable ha sido menos explorado. En la actualidad, la dieta de bajo índice glicémico sería probablemente más beneficiosa que otros tipos de dieta $^{23-26}$, debido a que produce una baja de peso con disminución especialmente del compartimiento graso, lo que permite mantener o mejorar la masa magra. Es interesante poner en evidencia que aún cuando las embarazadas con SOP tuvieron una ganancia de peso similar a las embarazadas sin SOP (11 a 12 kgs), no se logró prevenir que estas pacientes presentaran un mayor porcentaje diabetes gestacional $(35,2 \%$ en SOP y $5,0 \%$ en controles) y una mayor frecuencia de recién nacidos PEG en comparación a las mujeres normales $(10,0 \% v / s 2,8 \%)$ pese a que se mantuvieron las medidas no farmacológicas durante todo el embarazo. Además, las insulinemias fueron siempre mayores en las embarazadas con SOP en comparación a las embarazadas sin SOP.

Estudios longitudinales de tolerancia a la glucosa durante el embarazo normal, muestran un aumento progresivo de la insulina post estímulo, sin compromiso concomitante de la tolerancia a la glucosa, lo que revela el desarrollo de una insulino resistencia fisiológica progresiva durante el embarazo, lo cual se hace más evidente a partir del segundo trimestre ${ }^{27}$. De acuerdo a los datos del presente estudio, en las embarazadas con SOP se observó una exacerbación de este fenómeno, el cual no se corrigió en forma óptima con las medidas no farmacológicas y podría dar cuenta de la mayor frecuencia de diabetes gestacional observada en estas pacientes. El presente estudio además, permitió obtener valores de referencia para glicemias e insulinemias durante el embarazo en mujeres chilenas normales y con SOP, lo que a futuro permitirá un mejor control metabólico de estas mujeres.

Al momento del registro de los datos no había experiencia internacional suficiente para mantener el uso de metformina durante todo el embarazo, motivo por el cual el fármaco fue suspendido en forma progresiva al establecerse el diagnóstico de embarazo. Por tal motivo, el manejo de la IR durante el segundo y tercer trimestre del embarazo fue solamente en base a la dieta y el ejercicio factible de realizar según la condición obstétrica. Actualmente, el uso de metformina durante el embarazo es un tratamiento recomendado. La mantención de la terapia con metformina hasta el final del embarazo, ha demostrado ser eficaz en la prevención de abortos del primer trimestre, diabetes gestacional y sindrome hipertensivo del embarazo $^{28-32}$. Además, se asocia a menores concentraciones de andrógenos maternos ${ }^{8}$, lo que incidiría en el peso de nacimiento de los niños, ya que se 
ha demostrado una asociación entre los niveles de andrógenos maternos y el peso al nacer ${ }^{33}$. Estudios de seguimiento de los niños nacidos de madres con SOP tratadas con metformina durante todo el embarazo, no han demostrado alteraciones en el desarrollo pondo estatural ni sicomotor de estos niños 34,35

Por lo tanto, el uso de metformina en el embarazo, especialmente de la mujer con SOP tendría tres potenciales aplicaciones terapéuticas: disminuir el riesgo de abortos prematuros, disminuir el riesgo de patologías del embarazo y del período post-parto y disminuir el riesgo de androgenización prenatal ${ }^{36-44}$. Tomando en cuenta los antecedentes anteriormente descritos, y considerando que está ampliamente demostrado el efecto deletéreo que tienen las complicaciones del embarazo en el desarrollo fetal, la Asociación Americana de Diabetes en su reunión del año 2005, aceptó el uso de metformina durante todo el

\section{REFERENCIAS}

1. Ehrmann Da, Barnes RB, Rosenfield RL, Cavaghan MK, Imperial J. Prevalence of impaired glucose tolerance and diabetes in women with polycystic ovary syndrome. Diabetes Care 1999; 22: 141-6.

2. Legro RS, Kunselman AR, Dodson WC, Dunaif A. Prevalence and predictors risk for type 2 diabetes mellitus and impaired glucose tolerance in polycystic ovary syndrome: a prospective, controlled study in 254 affected women. J Clin Endocrinol Metab 1999; 84: 165-9.

3. Sir-Petermann T. Polycystic ovary syndrome, a pathway to type 2 diabetes. Nutrition 2005; 21 : 1160-3.

4. Urman B, Sarac E, Dogan L, Gurgan T. Pregnancy in infertile PCOD patients. Complications and outcome. J Reprod Med 1997; 42: 501-5.

5. Ibanez L, Potau $N$, Francois $I$, De Zegher $F$. Precocious pubarche, hyperinsulinism and ovarian hyperandrogenism in girls: relation to reduced fetal growth. J Clin Endocrinol Metab 1998; 83: 3558-62.

6. Sir-Petermann T, Hitschfeld C, Maliqueo M, Codner E, Echiburu B, Gazitua R, et al. Birth weight in offspring of PCOS mothers. Hum Reprod 2005; 20: $2122-6$ embarazo en pacientes de alto riesgo como pacientes con diabetes gestacional o pacientes diabéticas tipo 2 o portadoras de SOP que se embarazan.

En síntesis, las mujeres con SOP que logran un embarazo único de término presentan un porcentaje significativamente más alto de diabetes gestacional y una mayor frecuencia de niños pequeños para la edad gestacional que las embarazadas normales, a pesar de presentar una ganancia de peso similar a ellas. Estas complicaciones podrían estar relacionadas con el peso al inicio del embarazo o con una condición propia del SOP, ya que el control metabólico con dieta y ejercicio durante todo el embarazo no son suficientes para prevenir dichas complicaciones.

\section{Agradecimientos}

Los autores agradecen al personal paramédico del laboratorio por su abnegada labor.

7. Sir-Petermann T, Maliqueo M, Angel B, Lara He, Perez-Bravo F, Recabarren SE. Maternal serum androgens in pregnant women with polycystic ovary syndrome: possible implications in prenatal androgenization. Hum Reprod 2002; 10: 2573-9.

8. Glueck CJ, Goldenberg N, Wang P, Loftspring M, SHERman A. Metformin during pregnancy reduces insulin resistance, insulin secretion, weight, testosterone and development of gestational diabetes: prospective longitudinal assessment of women with polycystic ovary syndrome from preconception throughout pregnancy. Hum Reprod 2004a; 19: 510-21.

9. Kiddy DS, Hamilton-Fairley D, Bush A, Short F, ANYAOKU V, ReED MJ, et AL. Improvement in endocrine and ovarian function during dietary treatment of obese women with polycystic ovary syndrome. Clin Endocrinol (Oxf) 1992; 36: $105-11$.

10. Holte J, Bergh T, Berne C, Wide L, Lithell H. Restored insulin sensitivity but persistently increased early insulin secretion after weight loss in obese women with polycystic ovary syndrome. $J$ Clin Endocrinol Metab 1995; 80: 2586-93.

11. Moran LJ, Noakes M, Clifton PM, Tomlinson L, Galletly C, Norman RJ. Dietary composition in restoring reproductive and metabolic physiology 
in overweight women with polycystic ovary syndrome. J Clin Endocrinol Metab 2003; 88: 812-9.

12. Crosignani PG, Colombo M, Vegetti W, Somigliana E, Gessati A, Ragni G. Overweight and obese anovulatory patients with polycystic ovaries: parallel improvements in anthropometric indices, ovarian physiology and fertility rate induced by diet. Hum Reprod 2003; 18: 1928-32.

13. Tolino A, Gambardella V, Caccavale C, D’Ettore A, GiannotTi F, D'Anto V, ET AL. Evaluation of ovarian functionality after a dietary treatment in obese women with polycystic ovary syndrome. Eur J Obstet Gynecol Reprod Biol 2005; 119: 87-93.

14. The Rotterdam ESHRE/ASRM-Sponsored PCOS consensus workshop group. Revised 2003 consensus on diagnostic criteria and long-term health risks related to polycystic ovary syndrome (PCOS). Hum Reprod 2004; 19: 41-7.

15. Balen AH, Laven JS, Tan SL, Dewailly D. Ultrasound assessment of the polycystic ovary: international consensus definitions. Hum Reprod Update 2003; 9: 505-14.

16. American College of Obstetricians and Gynecologists. ACOG Practice Bulletin № 30. Gestational diabetes. Obstet Gynecol 2001; 98: 525-38.

17. Juez G, Lucero E, Ventura P, González H, Tapia Jl, Winter A. Crecimiento intrauterino en recién nacidos chilenos de clase media. Rev Chil Pediatr 1989; 60: 198-202.

18. Heard MJ, Pierce A, Carson SA, Buster JE. Pregnancies following use of metformin for ovulation induction in patients with polycystic ovary syndrome. Fertil Steril 2002; 77: 669-73.

19. Costello MF, Eden JA. A systematic review of the reproductive system effect of metformin in patints with polycystic ovary syndrome. Fertil Steril 2003; 79: 1-13.

20. Gracia R, Veloso D, Véliz C. Eficacia de un protocolo terapéutico en pacientes con síndrome de ovario poliquístico. Estudio prospectivo. Rev Chil Obstet Ginecol 2004; 69: 347-52.

21. Checa Ma, Requena A, Salvador C, Tur R, Callejo J, EsPinOs JJ, ET AL. Insulin-sensitizing agents: use in pregnancy and as therapy in polycystic ovary syndrome. Hum Reprod Update 2005; 11: 37590.

22. Thatcher SS, Jackson EM. Pregnancy outcome in infertile patients with polycystic ovary syndrome who were treated with metformin. Fertil Steril 2006; 85: 1002-9.
23. Reaven GM. The insulin resistance syndrome: definition and dietary approaches to treatment. Annu Rev Nutr 2005; 25: 391-406.

24. Mcauley KA, Smith KJ, Taylor RW, Mclay RT, Williams SM, Mann JI. Long-term effects of popular dietary approaches on weight loss and features of insulin resistance. Int J Obes (Lond) 2006; 30: 342-9.

25. Moran LJ, Noakes M, Clifton PM, Wittert GA, Williams G, Norman RJ. Short-term meal replacements followed by dietary macronutrient restriction enhance weight loss in polycystic ovary syndrome. Am J Clin Nutr 2006; 84: 77-87.

26. Krieger JW, Sitren HS, Daniels MJ, LangkampHenken B. Effects of variation in protein and carbohydrate intake on body mass and composition during energy restriction: a meta-regression 1. Am J Clin Nutr 2006; 83: 1442-3.

27. ButTe NF. Carbohydrate and lipid metabolism in pregnancy: normal compared with gestational diabetes mellitus. Am J Clin Nutr 2000; 71: 1256S61S.

28. Coetzee EJ, Jackson WP. Metformin in management of pregnant insulin-independent diabetics. Diabetologia 1979;16: 241-5.

29. CoetzeE EJ, JaCKson WP. The management of noninsulin-dependent diabetes during pregnancy. Diabetes Res Clin Pract 1986; 1: 281-7.

30. Glueck CJ, Goldenberg N, Streicher P, Wang P. Metformin and gestational diabetes. Curr Diab Rep 2003; 3: 303-12.

31. Tran Nd, Hunter $S_{k}$, Yankowitz J. Oral hypoglycemic agents in pregnancy. Obstet Gynecol Surv 2004; 59: 456-63.

32. Arias MC, Reid I, Acuña M, Munster H. Terapia con metformina y embarazo en pacientes con síndrome de ovario poliquístico. Rev Chil Obstet Ginecol 2004; 69: 290-3.

33. Carlsen SM, Jacobsen G, Romundstad P. Maternal testosterone levels during pregnancy are associated with offspring size at birth. Eur J Endocrinol 2006; 155: 365-70.

34. Glueck Cj, Goldberg N, Pranikoff J, Loftspring M, Sieve L, WANG P. Height, weight, and motor social development during the first 18 months of life in 126 infants born to 109 mothers with polycystic ovary syndrome who conceived on and continued metformina through pregnancy. Hum Reprod 2004b;19: 1323-30.

35. Glueck CJ, Salehi M, Sieve L, Wang P. Growth, motor, and social development in breast -and 
formula-fed infants of metformin-treated women with polycystic ovary syndrome. J Pediatr 2006; 148: 628-32.

36. JakUbowicz DJ, IUorno MJ, JakUbowicz S, ROBerts KA, Nestler JE. Effects of metformin on early pregnancy loss in the polycystic ovary syndrome. J Clin Endocrinol Metab 2002; 87: 524-9.

37. Glueck CJ, Wang P, Goldenderg N, Sieve-Smith L. Pregnancy outcomes among women with polycystic ovary syndrome treated with metformina. Hum Reprod 2002a; 17: 2858-64.

38. Glueck CJ, Wang P, Kobayashi S, Phillips H, SieveSMITH L. Metformin therapy throughout pregnancy reduces the development of gestational diabetes in women with polycystic ovary syndrome. Fertil Steril 2002b; 77: 520-5.

39. Norman RJ, Wang JX, Hague W. Should we continue or stop insulin sensitizing during pregnancy? Curr Opin Obstet Gynecol 2004; 16: 245-50.

40. Simmons D, Walters BN, Rowan JA, Mcintyre HD. Metformin therapy and diabetes in pregnancy. Med J Aust 2004; 180: 462-4.
41. Vanky E, Salvesen Ka, Heimstad R, Fougner KJ, ROMUNDSTAD P, CaRLSEN SM. Metformin reduces pregnancy complications without affecting androgen levels in pregnant polycystic ovary syndrome women: results of a randomised study. Hum Reprod 2004; 19: 1734-40.

42. Brock B, Smidt K, Ovesen P, Schmitz O, Rungby J. Is metformin therapy for polycystic ovary syndrome safe during pregnancy? Basic Clin Pharmacol Toxicol 2005; 96: 410-2.

43. Vanky E, Salvesen Ka, Hjorth-Hansen H, Bjerve K, CARLSEN SM. Beneficial effect of metformin on pregnancy outcome in women with polycystic ovary syndrome is not associated with major changes in C-reactive protein levels or indices of coagulation. Fertil Steril 2006; 85: 770-4.

44. Kovo M, Weissman A, Gur D, Levran D, Rotmensch S, Glezerman M. Neonatal outcome in polycystic ovarian syndrome patients treated with metformin during pregnancy. J Matern Fetal Neonatal Med 2006; 19: 415-9. 